\title{
Occult parametrial metastasis in operable cancer cervix: is a routine radical hysterectomy justified?
}

\author{
Tony Jose*, Sushil Chawla, Atul Seth
}

Department of Obstetrics and Gynecology, Armed Forces Medical College, Pune, Maharashtra India

Received: 31 May 2017

Accepted: 09 June 2017

\section{*Correspondence:}

Dr. Tony Jose,

E-mail: oncotango@gmail.com

Copyright: (C) the author(s), publisher and licensee Medip Academy. This is an open-access article distributed under the terms of the Creative Commons Attribution Non-Commercial License, which permits unrestricted non-commercial use, distribution, and reproduction in any medium, provided the original work is properly cited.

\section{ABSTRACT}

Background: Operable Ca cervix is treated by a Type III Radical hysterectomy(RH) with pelvic lymphadenectomy and comprises of a complex parametrial resection (PR) due to the possibility of occult parametrial metastasis(OPM) leading to high post-operative and delayed morbidity especially after adjuvant radiotherapy(RT). If risk factors for OPM were known, a less radical procedure could be performed in low risk patients thus minimizing the morbidity.

Methods: A retrospective study was carried out to evaluate the incidence of OPM in operated patients and to study the risk factors associated with OPM and explore the theoretical feasibility of doing a type I hysterectomy in low risk patients.

Results: The incidence of OPM was $16.1 \%$ in all stages (0\% in IA, $10.71 \%$ in IB1, 33.3\% in Stage IB2 and $18.8 \%$ in IIA1). Primary tumor diameter (PTD) and LNM were the most statistically significant risk factors for OPM. $90 \%$ of patients with OPM also had lymph node metastasis (LNM). In this study, all patients with OPM had other risk factors for adjuvant treatment.

Conclusions: Small size early stage tumors may be considered for less radical surgery in the absence of obvious intermediate risk factors. A node dissection with negative frozen section during surgery can finally select patients for a simple hysterectomy $(\mathrm{SH})$. More evidence from randomized trials are needed to evaluate the oncological safety of a less radical procedure and define the type of surgery to be done.

Keywords: Carcinoma cervix, Lymph nodal metastasis, Occult parametrial metastasis, Parametrial resection, Radical hysterectomy

\section{INTRODUCTION}

Early stages of Ca cervix (Stages IA to stage IIA1) are considered operable and is treated by Type III Radical Hysterectomy (RH) with bilateral pelvic lymphadenectomy especially in younger patients and is designed to surgically cure the disease. ${ }^{1}$ Radical hysterectomy typically involves the parametrial resection (PR) requiring extensive ureteric dissection resulting in hemorrhage and injuries to ureter and bladder leading to urinary fistulae. Pelvic autonomic nerves also get transected during PR leading to a host of physiological dysfunctions and morbidity (urogenital, rectal and sexual dysfunction). This is considered necessary because parametrium remains one of the common sites of spread, and parametrial metastasis (PM) is considered to be a high-risk prognostic factor in Ca cervix. Rates of PM varies from $6-13 \%$ in various studies and depends on many factors like tumor size, LVSI, Depth of stromal invasion (DOSI), and Pelvic LN metastasis (PLM)., ${ }^{2,3}$ Though cases with obvious PM undergo primary radiotherapy (RT), being a clinically staged disease, 
occult parametrial metastasis (OPM) is often missed even with advanced imaging like MRI only to be finally discovered at histological examination of the specimen. ${ }^{4}$ Thus, though radical surgery is done with curative intent in early ca cervix, definitive confirmation of clear parametria can only be obtained by a meticulous grossing and careful histological study of the operative specimen for OPM. OPM warrants adjuvant RT being a high-risk factor and this increases the morbidity of RH.

The maximum morbidity of $\mathrm{RH}$ being due to parametrial resection, several studies were done to explore the possibility of minimizing the extent of parametrial resection thereby reducing morbidity.,6 This was based on the premise that PM does not occur in the absence of certain high-risk factors (Tumor size, LVSI, Deep stromal invasion (DSI), PLM) and identification of a low risk subgroup of patients would enable a less radical procedure, even a simple hysterectomy to replace $\mathrm{RH}^{7,8}$ This would not only reduce the morbidity but also ensure wider availability of this less radical surgical option in resource starved environments with limited availability of expertise to perform a RH. However, sceptics oppose this concept based on the evidence from some studies that suggest that PM occurs in both medial and lateral parametrium independently and may not be predictable on the basis of risk factors. ${ }^{2}$ The aim of this study was to retrospectively find the incidence of occult parametrial metastasis in operable stages of Ca cervix (Stages IA2 to Stage II A1), study the potential risk factors for parametrial metastasis (PM) that can pre-operatively predict OPM and also explore the role of a simple hysterectomy $(\mathrm{SH})$ in place of a $\mathrm{RH}$ in such low risk patients.

\section{METHODS}

This retrospective study included all patients clinically staged to be Stage IA1 (with LVSI) to Stage IIA1 Ca cervix who underwent $\mathrm{RH}$ with pelvic lymph node dissection (RH) in histologically confirmed squamous cell carcinoma (SCC), Adeno-squamous (ASC) and adenocarcinomas (AC) in a tertiary care hospital for a period of 4 years. Pre-operative evaluation included clinical staging, which included permitted staging investigations including colposcopy to rule out extension to vagina wherever indicated. $\mathrm{RH}$ included ligating uterine artery at its origin, dissection of the entire course of the ureter up to its entry into the bladder, and resection of the cardinal and uterosacral ligaments as laterally as possible along with para-colpos and $1 \mathrm{~cm}$ of disease-free cuff of vagina.

Pelvic lymphadenectomy included systematic clearance of all node bearing fatty tissue laterally till the genitofemoral nerve, medially till the internal iliac artery, superiorly till the mid common iliac arteries, inferiorly till the circumflex iliac vessels and posteriorly till the obturator nerve (internal iliac, external iliac, obturator groups of lymph nodes). The resected specimen was subjected to standard grossing and sampling techniques. Primary tumor diameter (PTD) and node count was measured during grossing and histology, tumor grade, lymph vascular space invasion (LVSI), DOSI and OPM were recorded during microscopic examination. OPM was defined as either direct tumor extension, parametrial lymph nodal metastasis or presence of tumor emboli in the parametrium. Patients were administered adjuvant RT as indicated by the high risk (Nodal metastasis, PM and positive vaginal margins) and intermediate risk factorsany 2 or more (Tumor $>2 \mathrm{~cm}$, deep stromal invasion (DSI) and LVSI). ${ }^{9}$ Data was analyzed to study PM in different stages and sub-stages and was correlated with the risk factors.

\section{RESULTS}

During the study period, 202 patients were diagnosed to have ca cervix on biopsy and 64 were clinically staged as operable (Stage I A- 8, Stage IB1-28, Stage I B2-15, Stage II A1- 11) and were planned for RH. 62 patients completed the procedure ( $\mathrm{RH}$ not done in two cases - one due to positive pelvic LN metastasis on frozen section (FS) and one due to bladder infiltration). Table 1 shows the demographic and pathologic characteristics of the study group. Out of the 62 patients who were operated, 28 patients received adjuvant RT, 23 for lymph nodal metastasis (LNM) [out of which 9 also had occult parametrial metastasis (OPM)] and 5 patients for presence of two or more intermediate risk factors (LVSI, PTD $>2 \mathrm{~cm}, \mathrm{DSI})$.

Table 1: Demographic characteristics and pathologic risk factors.

\begin{tabular}{|c|c|}
\hline Variable & All subjects $(n=62)$ No $(\%)$ \\
\hline \multicolumn{2}{|l|}{ Age (years) } \\
\hline Median (range) & $53.04(38-73)$ \\
\hline \multicolumn{2}{|l|}{ Tumor size $(\mathrm{cm})$} \\
\hline Median (range) & $2.25(0.4-7.4)$ \\
\hline \multicolumn{2}{|l|}{ Histologic Type } \\
\hline Squamous & $50(80.6)$ \\
\hline Adenocarcinoma & $8(12.9)$ \\
\hline Adenosquamous & $4(6.5)$ \\
\hline \multicolumn{2}{|l|}{ Stage (FIGO) } \\
\hline IA1 with LVSI & $4(6.5)$ \\
\hline IA2 & $4(6.5]$ \\
\hline IB1 & $28(45.1)$ \\
\hline IB2 & $15(24.2)$ \\
\hline IIA1 & $11(17.7)$ \\
\hline \multicolumn{2}{|c|}{ Depth of invasion (DOI) } \\
\hline$<50 \%$ & $35(56.4)$ \\
\hline$>50 \%$ & $27(43.6)$ \\
\hline LVSI +ve & $34(54.8)$ \\
\hline Positive PLN & $23(37)$ \\
\hline OPM & $10(16.1)$ \\
\hline $\begin{array}{l}\text { Positive margins } \\
\text { (vaginal) }\end{array}$ & 0 \\
\hline
\end{tabular}


10 patients had OPM in the study group with an incidence of $16.1 \%$ in the operable stages 9 of whom also had LNM $(90 \%)$. These patients included 3 in stage IB1 $(3 / 28), 5$ in I B2 $(5 / 15)$ and 2 in stage IIA1(2/11) the incidences being $10.71 \%, 33.3 \%$ and $18.18 \%$ respectively in these stages. PLM was studied in the two groups while $90 \%$ patients with OPM had PLM, only $26.9 \%$ showed PLM in the non OPM group, a difference that was statistically significant. The other potential risk factors were also compared between the OPM subgroup and the non OPM subgroup. The median (PTD) in OPM group was $4.50(2.5-7.4) \mathrm{cm}[3.24 \mathrm{~cm}$ in I B1, 6.18 in IB2 and 3.45 in IIA1] as against $2.15(0.6-7) \mathrm{cm}$ in the group that did not have OPM the difference being statistically significant thus indicating a clear relationship with tumor size. In the OPM group, while $80 \%$ had LVSI, $70 \%$ had DSI as against $48 \%$ and $50 \%$ in the group without OPM but none of these was found to be significant. The potential risk factors are summarized in Table 2.

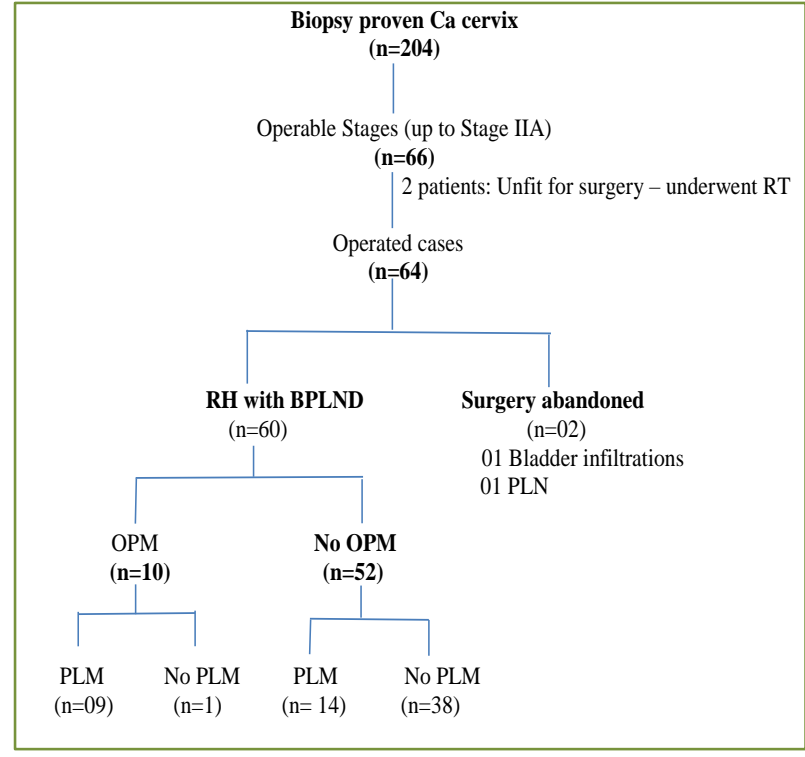

Figure 1: Study design.

Table 2: Risk factors for OPM.

\begin{tabular}{|c|c|c|c|}
\hline Variable & No. PM group $(\mathrm{n}=52)$ No. $(\%)$ & OPM group $(\mathrm{n}=10)$ No. $(\%)$ & P value \\
\hline \multicolumn{4}{|l|}{ Age (years) } \\
\hline Median (range) & $55(38-73)$ & $47(40-72)$ & 0.08 \\
\hline \multicolumn{4}{|l|}{ Tumor size (cm) } \\
\hline Median (range) & $2.15(0.6-7)$ & $4.50(2.5-7.4)$ & 0.002 \\
\hline \multicolumn{4}{|l|}{ Histologic type } \\
\hline Squamous & $43(69.35)$ & $7(70)$ & \\
\hline Adenocarcinoma & $7(11.29)$ & $2(20)$ & \\
\hline Adenosquamous & $2(3.2)$ & $1(10)$ & \\
\hline \multicolumn{4}{|l|}{ Stage (FIGO) } \\
\hline IA1 with LVSI & $4(6.45)$ & 0 & \\
\hline IA2 & $4(6.45)$ & 0 & \\
\hline IB1 & $24(38.7)$ & $3(30)$ & \\
\hline IB2 & $10(19.2)$ & $5(50)$ & \\
\hline IIA1 & $9(17.3)$ & $2(20)$ & \\
\hline \multicolumn{4}{|l|}{ Depth of invasion (DOI) } \\
\hline$<50 \%$ & $32(61.5)$ & $4[40]$ & \multirow[t]{2}{*}{0.206} \\
\hline$>50 \%$ & $20(38.5)$ & $6[60]$ & \\
\hline \multicolumn{4}{|l|}{ LVSI } \\
\hline+ ve & $25(48)$ & $8(80]$ & \multirow[t]{2}{*}{0.08} \\
\hline -ve & $27(52)$ & $2(20)$ & \\
\hline \multicolumn{4}{|l|}{ PLN } \\
\hline Positive & $14(26.9)$ & $9(90)$ & \multirow{2}{*}{0.0003} \\
\hline Negative & $38(73.1)$ & $1(20)$ & \\
\hline Positive margins (vaginal) & 0 & 0 & \\
\hline
\end{tabular}

\section{DISCUSSION}

Management of operable ca cervix is slowly shifting towards tailored less radical approaches not only aimed at reducing post-operative morbidity but also to consider fertility preservation in younger patients when possible within the limits of oncological safety. One of the main strategies to reduce morbidity is to reduce the radicality 
of PR by identifying a low risk subgroup where the risk of OPM is low. Covens et al suggested tumor size $<2 \mathrm{~cm}$ and grade of the tumor as predictors of negative PM. Winter et al and Puente et al proposed lymph nodal status as an indicator of OPM and recommended routine frozen section(FS) of lymph nodes prior to $\mathrm{RH}$ and parametrial resection. ${ }^{5,7,8}$ However, Benedetti-Panici et al in their study of patterns of parametrial involvement and LN metastasis found that lateral parametrium was the most common site of PM and that too by tumor embolization and hence OPM could occur independently and unpredictably in both lateral and medial parametrium. This led sceptics to believe that entire parametrium needs to be routinely removed irrespective of other factors though Puente et al has contradicted this finding in their study. ${ }^{2,7}$ It has also been debated whether taking a chance with oncological safety is going to benefit the patient significantly? The entire purpose of limiting the radicality of parametrial resection is to avoid possible long-term complications (Fistulae, bladder dysfunction, anal dysfunction and sexual dysfunction) that occur due to visceral damage and injuries to autonomic nerves and the complications of fibrosis and endarteritis especially after adjuvant RT. It also reduces operating time, blood loss, anesthesia complications and hastens immediate post op recovery. Nevertheless it is to be remembered that the risk of fistulas is low $(<1 \%)$ in skilled hands and one should consider less radical surgery only when the risk of PM is low enough to justify the advantage of limited resection without compromising oncological safety. ${ }^{10}$ Presently there are no randomized studies addressing the issue of oncological safety of less radical procedure except for the study by Landoni et al which demonstrated reduced post op morbidity of Type II vs Type III Rh in selected early stage ca cervix without compromising on the outcomes and survival. ${ }^{11}$

Notwithstanding the findings of this study, PM remains an independent high-risk factor and patients with OPM fare poorly even after administration of adjuvant RT. ${ }^{8}$ Hence it would be worthwhile to look at the risk factors associated with OPM in Ca cervix. Chang et al evaluated the potential risk factors for PM in stage B1 cervical cancers and found that tumor size $>2 \mathrm{~cm}$, LVSI, DSI and PLM increased chances of PM out of which PTD $>3 \mathrm{~cm}$ and PLM were independent risk factors. ${ }^{12}$ Sevin et al also found significantly lower rates of PLM in stages I and II of cervical cancers when the PTD was less than $2 \mathrm{~cm}$ and the rates doubled when it reached $3 \mathrm{~cm} .{ }^{13}$ The findings of our study is also in agreement with all these findings and PLM and PTD have been found to be the most significant risk factors for OPM. However, these criteria are based on the final histopathology and hence cannot be preoperatively assessed (except for PTD) thus making prediction of OPM risk impossible. Pre-op MRI has been tried to study both the tumor size and depth of invasion in the absence of obvious radiologically detectable PM in various studies and it has been found that MRI assessed tumor size and depth of stromal invasion is a reliable predictor for OPM. ${ }^{14}$ Squamous cell carcinoma antigen
(SCCAg) was studied as a tumor marker for detecting high risk tumors and Chang et al has shown a SCCAg value $>1.40 \mathrm{ng} / \mathrm{ml}$ to be a reliable indicator of $\mathrm{PM}$ especially when combined with PTD $>3 \mathrm{~cm}$ as a second parameter in their prediction model. ${ }^{12}$ They however recommended a nerve sparing $\mathrm{RH}$ as the less radical procedure in view of the unpredictable nature of parametrial metastasis. LVSI and DSI are two important risk factors that are often linked to PM and PLM and a routine punch biopsy cannot conclusively comment on either. LEEP and conization specimens were also evaluated for definitive pre-operative triage based on LVSI and DOSI but even in small sized tumors only when the excision margins were negative could negative LVSI and shallow DOSI predict low risk for PM and PLM. ${ }^{15}$ Status of pelvic lymph nodes a factor strongly associated with risk of PM also cannot be emphatically ruled out even by the best available pre-op imaging including PET CT scans. Pluta et al in a pilot study used sentinel node evaluation with frozen section during $\mathrm{RH}$ to decide on the radicality of parametrial dissection and found no increase in recurrences in patients who after negative FS were assigned to extra-fascial hysterectomy with PLND after a mean 47 months follow up. ${ }^{16}$ However, PM was not evaluated in this study.

The findings of the present study indicate that the risk of OPM is definitely associated with LNM and PTD and this association is statistically significant. It is also clear that in all probability, most patients with OPM are likely to have LNM also. There is also a possible relationship between OPM with LVSI and DSI though this study did not show a statistically significant association possibly due to small numbers. Hence it can be inferred that a patient with small PTD where LVSI is absent and DOSI is $<50 \%$ are potential candidates for simple hysterectomy if preceded by a PLD with negative FS report on the nodes.

This would avoid the complications and morbidity of PR without compromising the oncological safety. If PR had not been performed in these patients who happened to have OPM in this study, 9 of these patients would have received adjuvant RT for LNM as would have the patient without LNM due to the presence of three intermediate risk factors. Thus, all these 10 patients would have received adjuvant treatment as indicated without the additional morbidity of RH. However, the implications of the oncologically inferior Type I hysterectomy on the risk of recurrence and long-term survival is a matter of speculation and has not been looked into in this study and has not been prospectively studied in literature.

Though there appears to be a genuine case for considering less radical surgeries including simple hysterectomy with PLND in low risk patients, what exactly is the low risk population still needs to be defined with a higher level of evidence. We need to await the results of ongoing prospective studies (GOG 278, Con Cerv and SHAPE trials) and also need to conduct well 
designed randomized trials comparing simple hysterectomy with with RH both combined with PLND in selected patients considered low risk for PI.

\section{ACKNOWLEDGMENTS}

Authors would like to thank Dr. Shakti Vardhan, Professor and HOD, Department of Obstetrics and Gynecology for the guidance and HOD, Department of Pathology for all assistance in reviewing the HPR reports.

Funding: No funding sources

Conflict of interest: None declared

Ethical approval: The study was approved by the Institutional Ethics Committee

\section{REFERENCES}

1. Piver MS, Ghomi A. The twenty-first century role of Piver-Rutledge type III radical hysterectomy and FIGO stage IA, IB1, and IB2 cervical cancer in the era of robotic surgery: a personal perspective. J Gynecol Oncol. 2010;21:219-24.

2. Benedetti-Panici P, Maneschi F, D'Andrea F, Cutillo G, Rabittti C, Congiu M, et al. Early cervical carcinoma: the natural history of lymph node involvement redefined on the basis of thorough parametrectomy and giant section study. Cancer. 2000;88(10):2267-74.

3. Landoni F, Bocciolone L, Perego P, Maneo A, Bratina G, Mangioni C. Cancer of the cervix, FIGO stages IB and IIA: patterns of local growth and paracervical extension. Int $\mathbf{J}$ Gynecol Cancer. 1995;5(5):329-34.

4. Iwata S, Joja I, Okuno K, Miyagi Y, Sakaguchi Y, Kudo T, et al. Cervical carcinoma with full-thickness stromal invasion: efficacy of dynamic MR imaging in the assessment of parametrial involvement. Radiat Med. 2002;20(5):247-55.

5. Covens A, Rosen B, Murphy J, Laframboise S, DePetrillo D, Lickrish G, et al. How important is removal of the parametrium at surgery for carcinoma of the cervix? Gynecol Oncol. 2002;84(1):145-9.

6. Kinney WK, Hodge DO, Egorshin EV, Ballard DJ, Podratz KC. Identification of a low-risk subset of patients with stage IB invasive squamous cancer of the cervix possibly suited to less radical surgical treatment. Gynecol Oncol. 1995;57(1):3-6.

7. Puente R, Guzman S, Isreal E, Poblete MT. Do the pelvic lymph nodes predict the parametrial status in cervical cancer stages IB-IIA? Int J Gynecol Cancer. 2004;14(5):832-40.
8. Winter R, Haas J, Reich O, Koemetter R, Tamussino $\mathrm{K}$, Lahousen $\mathrm{M}$, et al. Parametrial spread of cervical cancer in patients with negative pelvic lymph nodes. Gynecol Oncol. 2002;84(2):252-7.

9. Carballo N, González-Cortijo L, González-Martín A, Rojo A, Chiva L. Indications for adjuvant radiotherapy treatment after surgery and novel modalities for treatment. Gynecol Oncol. 2008;110(3 Suppl 2):S41-4.

10. Steed H, Capstick V, Schepansky A, Honore L, Hiltz M, Faught W . Early cervical cancer and parametrial involvement: Is it significant? Gynecol Oncol. 2006;103:53-7.

11. Landoni F, Maneo A, Cormio G, Perego P, Milani R, Caruso $\mathrm{O}$, et al. Class II versus class III radical hysterectomy in stage Ib-IIa cervical cancer: a prospective randomised study. Gynecol Oncol. 2001;80:3-12.

12. Chang SJ, Bristow RE, Ryu HS. A model for prediction of parametrial involvement and feasibility of less radical resection of parametrium in patients with FIGO stage IB1 cervical cancer. Gynecol Oncol. 2012;126:82-6.

13. Sevin BU, Nadji M, Lampe B, Lu Y, Kilsenbeck S, Koechli OR, et al. Prognostic factors of early stage cervical cancer treated by radical hysterectomy. Cancer. 1995;76:1978-86.

14. Kamimori T, Sakamoto K, Fujiwara K, Umayahara $\mathrm{K}$, Sugiyama $\mathrm{Y}$, Utsugi $\mathrm{K}$, et al. Parametrial involvement in FIGO stage IB1 cervical carcinoma diagnostic impact of tumor diameter in preoperative magnetic resonance imaging. Int $\mathrm{J}$ Gynecol Cancer. 2011;21:349-54.

15. Smith B, Georgia A, Phillips MG, Backes FJ, O'Malley DM, Cohn DE, et al. Less radical surgery for early-stage cervical cancer: Can conization specimens help identify patients at low risk for parametrial involvement? Gynecol Oncol. 2017;144:290-3.

16. Pluta M, Rob L, Charvat M, Chmel R, Halaska M, Skapa $\mathrm{P}$,et al. Lessradical surgery than radical hysterectomy in early stage cervical cancer - a pilot study. Gynecol Oncol. 2009;113:181-4.

Cite this article as: Jose T, Chawla S, Seth A. Occult parametrial metastasis in operable cancer cervix: is a routine radical hysterectomy justified? Int J Reprod Contracept Obstet Gynecol 2017;6:2874-8. 